CTP-TAMU-52/92

hep-ph@xxx/9207202

\title{
Dilatonic Domain Walls
}

\author{
HoSeong La* \\ Center for Theoretical Physics \\ Texas A\&M University \\ College Station, TX 77843-4242, USA
}

\begin{abstract}
A static domain wall with infinitesimal thickness is obtained in the theory of a scalar field coupled to gravity with the dilaton, i.e. the Jordan-Brans-Dicke gravity. The value of the dilaton is determined in terms of the Brans-Dicke parameter $\omega$. In particular the metric for the wall becomes flat and the dilaton, though nontrivial, has vanishing kinetic energy (i.e. $\omega=0$ ) so that there is no gravitational effect due to such a dilatonic domain wall. Some remarks on possible thick domain walls are given too.

PACS numbers: 04.60.+n, 04.50.+h, 98.80.-k, 11.10.-z, 11.30.Er, 11.17.+y
\end{abstract}

06/92

* e-mail address: hsla@phys.tamu.edu, hsla@tamphys.bitnet 
It is interesting to observe the fact that most of gravitational theories known so far allow extended objects as their classical solutions[1] [2], although the chances of actual existence are not necessarily high. Nevertheless, the properties of such extended objects can often provide constraints on various aspects of phenomenological implications of the theories. Theories which undergo phase transition via spontaneous symmetry breaking are particularly interesting because they become building blocks of cosmological models and often provide topological defects, e.g. monopoles, cosmic strings, domain walls, etc. [1]. Such defects in principle can be formed in the early universe 3 .

In this letter we shall investigate the structure of (infinitesimally thin) domain wall solutions in the Jordan-Brans-Dicke (JBD) theory, which is a gravitational theory with the dilaton in the four-dimensional space-time. From the string theory's point of view, the JBD theory with a specific Brans-Dicke (BD) parameter is a natural effective gravitational theory before the dilaton freezes up. Furthermore, a cosmological model can also be built based on the JBD theory [4]. Thus it is worth while to investigate the existence of extended objects in this context. Cosmic string solutions in this theory were previously studied in ref. $[5]$.

We shall consider the action for the JBD theory

$$
\mathcal{S}=\int d^{4} x \sqrt{-\widetilde{g}} \mathrm{e}^{-2 \widetilde{\phi}}\left(\widetilde{R}-4 \omega \partial_{\mu} \widetilde{\phi} \partial^{\mu} \widetilde{\phi}\right)+\mathcal{S}_{M}\left[\widetilde{g}_{\mu \nu}\right]
$$

where $\omega$ is the BD parameter of the theory. In particular the $\omega=-1$ case corresponds to the action of the dilaton gravity from string theory [6]. For our purpose we take $\mathcal{S}_{M}$ as the action for the real scalar field with a double well potential:

$$
\mathcal{S}_{M}=-\frac{1}{2} \int d^{4} x \sqrt{-\widetilde{g}}\left(\partial_{\mu} \Phi \partial^{\mu} \Phi+\lambda\left(\Phi^{2}-\eta^{2}\right)^{2}\right)
$$

$\mathcal{S}_{M}$ in particular has a discrete symmetry $\Phi \rightarrow-\Phi$. In the static case, this discrete symmetry is equivalent to the time reversal symmetry. This is why the domain wall structure is related to the $\mathrm{CP}$ phases $[7][$. For convenience we redefine the variables as

$$
\omega=\frac{1}{4 \beta^{2}}-\frac{3}{2}, \quad \mathrm{e}^{-2 \widetilde{\phi}}=\frac{1}{16 \pi G} \mathrm{e}^{-2 \beta \phi}, \quad \widetilde{g}_{\mu \nu}=\mathrm{e}^{2 \beta \phi} g_{\mu \nu},
$$

then the action now becomes

$$
\mathcal{S}=\frac{1}{16 \pi G} \int d^{4} x \sqrt{-g}\left(R-\partial_{\mu} \phi \partial^{\mu} \phi\right)+\mathcal{S}_{M}\left[\mathrm{e}^{2 \beta \phi} g_{\mu \nu}\right]
$$


Setting $\phi=0$, this action reduces to that of a real scalar field coupled to the Einstein gravity, in which case a static thin domain wall solution is known to exist, although it is gravitationally unstable [9]. In this letter we shall present a stable domain wall, which is a static classical solution of eq.(1). In our case although $\widetilde{g}_{\mu \nu}$ is a physical metric of the space-time, eq. (4) is very convenient for practical purposes. Although $\widetilde{\phi}$ is the actual dilaton, we will call $\phi$ a dilaton too since they are related by a simple field redefinition.

By varying eq.(四) with respect to the new metric $g_{\mu \nu}$, we obtain

$$
R_{\mu \nu}=\partial_{\mu} \phi \partial_{\nu} \phi+8 \pi G\left(T_{\mu \nu}-\frac{1}{2} g_{\mu \nu} T\right)
$$

where the "energy-momentum" tensor is given by

$$
T_{\mu \nu}=-\frac{2}{\sqrt{-g}} \frac{\delta \mathcal{S}_{M}}{\delta g^{\mu \nu}}=-\frac{1}{2}\left[\mathrm{e}^{2 \beta \phi} g_{\mu \nu} g^{\alpha \beta} \partial_{\alpha} \Phi \partial_{\beta} \Phi-2 \mathrm{e}^{2 \beta \phi} \partial_{\mu} \Phi \partial_{\nu} \Phi+g_{\mu \nu} \mathrm{e}^{4 \beta \phi} \lambda\left(\Phi^{2}-\eta^{2}\right)^{2}\right]
$$

Note that $T_{\mu \nu}$ is not conserved due to the dilatonic contribution. The physical energymomentum tensor $\widetilde{T}_{\mu \nu}^{\text {matter }}+\widetilde{T}_{\mu \nu}^{\widetilde{\phi}}$ satisfies the gravitational equation of motion for JBD theory 10],

$$
\widetilde{R}_{\mu \nu}-\frac{1}{2} \widetilde{g}_{\mu \nu} \widetilde{R}=8 \pi \mathrm{e}^{2 \widetilde{\phi}}\left(\widetilde{T}_{\mu \nu}^{\text {matter }}+\widetilde{T}_{\mu \nu}^{\widetilde{\phi}}\right)
$$

where $\nabla^{\mu} \widetilde{T}_{\mu \nu}^{\text {matter }}=0$ and

$$
\begin{aligned}
\widetilde{T}_{\mu \nu}^{\widetilde{\phi}}= & \frac{1}{8 \pi} \mathrm{e}^{-2 \widetilde{\phi}}\left(2 \partial_{\mu} \partial_{\nu} \widetilde{\phi}+2 \widetilde{\Gamma}_{\mu \nu}^{\alpha} \partial_{\alpha} \widetilde{\phi}+\frac{1}{2} \mathrm{e}^{2 \widetilde{\phi}} \widetilde{g}_{\mu \nu} \widetilde{\square}^{2} \mathrm{e}^{-2 \widetilde{\phi}}\right) \\
& -\frac{\omega}{2 \pi} \mathrm{e}^{-2 \widetilde{\phi}}\left(\partial_{\mu} \widetilde{\phi} \partial_{\nu} \widetilde{\phi}-\frac{1}{2} \widetilde{g}_{\mu \nu} \partial^{\alpha} \widetilde{\phi} \partial_{\alpha} \widetilde{\phi}\right) .
\end{aligned}
$$

The second term of eq.(8) is proportional to $\omega$ so that it vanishes if $\omega=0$, but the first term is independent of $\omega$.

The field equations for the dilaton $\phi$ is

$$
\square^{2} \phi=\frac{1}{\sqrt{-g}} \partial_{\mu}\left(\sqrt{-g} g^{\mu \nu} \partial_{\nu}\right) \phi=-8 \pi G \beta T
$$

and the scalar field satisfies

$$
\partial_{\mu}\left(\sqrt{-g} \mathrm{e}^{2 \beta \phi} g^{\mu \nu} \partial_{\nu}\right) \Phi-2 \lambda \sqrt{-g} \mathrm{e}^{4 \beta \phi} \Phi\left(\Phi^{2}-\eta^{2}\right)=0
$$

In general, domain wall solutions are obtained in theories where a discrete symmetry is spontaneously broken. Note that the action eq.(2) for the matter field $\Phi$ has a discrete 
symmetry $\Phi \rightarrow-\Phi$ so that we can look for domain walls, when this symmetry is spontaneously broken. In the case where domain walls have infinitesimal thickness, we can approximate the wanted scalar field to behave as

$$
\Phi(z)= \begin{cases}\eta & \text { if } z>0 \\ -\eta & \text { if } z<0\end{cases}
$$

Then we are interested in the the domain wall which separates a space of the $\Phi=\eta$ phase from a space of the $\Phi=-\eta$ phase. Such an approximation is in fact reasonable for the cases where the Compton wavelength of the test particle is much longer than the thickness of the wall.

We shall try the following ansatz for domain wall solutions [11] [9]:

$$
d s^{2}=A(|z|)\left(-d t^{2}+d z^{2}\right)+B(|z|)\left(d x^{2}+d y^{2}\right) .
$$

Note that we have required the reflection symmetry between each side of the wall, which is an infinite plane perpendicular to the $z$-direction at $z=0$. In fact it turns out that this is quite a general ansatz. Normally $|z|$ is not analytic at $z=0$, but we can use the following prescription for $|z|$ to avoid such a difficulty:

$$
|z|=z[\theta(z)-\theta(-z)]
$$

where $\theta(z)$ is a step function defined by $\theta(z)=1$ for $z \geq 0, \theta(z)=0$ for $z<0$. Then $\partial_{z}|z|=[\theta(z)-\theta(-z)]+2 z \delta(z)$. If we promise that $\partial_{z}|z|$ shall be multiplied with some function of $z$ that does not have a pole at $z=0$, we can safely use an identification $\partial_{z}|z| \equiv \theta(z)-\theta(-z)$. Similarly, $\partial_{z}^{2}|z| \equiv 2 \delta(z)$. The reason we try to be careful about such analyticity is to check the consistency of the solutions at the wall, which turns out to be important to provide interesting constraints on the solutions.

Using this ansatz the field equations become

$$
\begin{aligned}
\left(\frac{A^{\prime}}{A}\right)^{\prime}+\frac{A^{\prime} B^{\prime}}{A B} & =-8 \pi G \lambda A \mathrm{e}^{4 \beta \phi}\left(\Phi^{2}-\eta^{2}\right)^{2}, \\
\left(\frac{B^{\prime}}{B}\right)^{\prime}+\frac{B^{\prime 2}}{B^{2}} & =-8 \pi G \lambda A \mathrm{e}^{4 \beta \phi}\left(\Phi^{2}-\eta^{2}\right)^{2}, \\
\frac{A^{\prime} B^{\prime}}{A B}-\left(\frac{A^{\prime}}{A}\right)^{\prime}-2 \frac{B^{\prime \prime}}{B}+\frac{B^{\prime 2}}{B^{2}} & =2 \phi^{\prime 2}+8 \pi G\left(2 \mathrm{e}^{2 \beta \phi} \Phi^{\prime 2}+\lambda A \mathrm{e}^{4 \beta \phi}\left(\Phi^{2}-\eta^{2}\right)^{2}\right), \\
\frac{B^{\prime}}{B} \phi^{\prime}+\phi^{\prime \prime} & =8 \pi G \beta\left(\mathrm{e}^{2 \beta \phi} \Phi^{\prime 2}+2 \lambda A \mathrm{e}^{4 \beta \phi}\left(\Phi^{2}-\eta^{2}\right)^{2}\right), \\
0 & =\left(B \mathrm{e}^{2 \beta \phi} \Phi^{\prime}\right)^{\prime}-2 \lambda A B \mathrm{e}^{4 \beta \phi} \Phi\left(\Phi^{2}-\eta^{2}\right),
\end{aligned}
$$


For thin walls we have $\Phi^{2}=\eta^{2}$ and $\Phi^{\prime}=0$ away from $z=0$ so that we shall first solve the above equations away from $z=0$, then shall check the consistency at the wall. Solving eqs. $(13 a-d)$ for $z \neq 0$, we obtain

$$
\begin{aligned}
& A(z)=(1+\widetilde{\kappa}|z|)^{\alpha^{2}-\frac{1}{2}} \\
& B(z)=(1+\widetilde{\kappa}|z|) \\
& \phi(z)=\alpha \ln (1+\widetilde{\kappa}|z|)
\end{aligned}
$$

where $\widetilde{\kappa}$ and the parameter $\alpha$ are yet to be determined, while the value of the dilaton at $z=0$ is taken to be zero.

Now let us check the consistency of the solution at the wall. Using the analytic property eq.(12) we prescribed, eq.(13b) reduces to

$$
\widetilde{\kappa} 2 \delta(z)=8 \pi G \lambda\left(\Phi^{2}-\eta^{2}\right)^{2},
$$

which leads to $\widetilde{\kappa}>0$. Similarly, eq.(13a) reduces to

$$
\left(\alpha^{2}-\frac{1}{2}\right) \widetilde{\kappa} 2 \delta(z)=8 \pi G \lambda\left(\Phi^{2}-\eta^{2}\right)^{2},
$$

which together with eq.(15) implies that

$$
\alpha^{2}=3 / 2 \text { so that } A=B
$$

This is a very strong constraint, which even the Einstein gravity case cannot satisfyl. Note that $\widetilde{\kappa}$ and $\eta$ have mass dimensions and the Newton's gravitational constant $G$ has inverse mass square dimension, while $\lambda$ is a dimensionless coupling constant. Using a dimensional analysis for a possible thick wall, if we have $G \lambda \eta^{4} \gg \widetilde{\kappa} \frac{\eta}{\sqrt{\lambda}}$ and $\Phi(z=0)=0$, the LHS of eqs.15)(16) are effectively comparable to the RHS by smearing out the delta function Thus this is a good approximate solution and the condition in fact corresponds to the weak gravitational field limit.

Eqs. $13 c, d)$ in turn require that

$$
\left(\Phi^{\prime}\right)^{2}=\frac{\widetilde{\kappa}}{4 \pi G} \delta(z)
$$

${ }^{1} \alpha=0$ corresponds to the Einstein gravity case. This also confirms that there is no static (stable) thin domain wall in this case. Note that this does not imply that there is no domain wall solution in the Einstein gravity. It is known that there exits a time-dependent thin wall solution: A. Vilenkin, Phys. Lett. 133B (1983) 177; J. Ipser and P. Sikivie, Phys. Rev. D30 (1984)712. 
and

$$
2 \beta \alpha^{2}+\alpha=0
$$

Thus $2 \alpha \beta+1=0$ and that eq. $(13 \theta)$ is also satisfied. For $\alpha^{2}=3 / 2, \beta^{2}=1 / 6$, which leads to the BD parameter $\omega=0$ for eq.(3).

Finally, $\widetilde{\kappa}$ can be determined from the "energy" density as follows: Using eqs.(15)-(18) we can compute the "energy-momentum" tensor eq.(6) as

$$
T_{\mu \nu}=\frac{\widetilde{\kappa}}{4 \pi G} \delta(z) \operatorname{diag}(1,-1,-1,0), \quad \widetilde{\kappa}>0 .
$$

Note that this is not the physical energy-momentum in eq.(7) so that getting negative "energy" density does not imply the instability of the wall.

Here we would like to call the readers attention to the fact that we have differential equations with the Dirac delta-function. Some may find that this is unreasonable because after all the Dirac delta-function is not a function but a distribution. But this is not completely unreasonable in field theory when we often need to be careful about the analyticity. The main intention is not to solve the differential equations in question but to check the consistency between equations. In this sense this is a sufficiently good approximation. In fact one can be more careful about this situation and can introduce distributional energy-momentum tensor in terms of delta-function from the beginning[12]. The result however is more or less equivalent because we also have derived the distributional "energymomentum" tensor using our prescription eq.(12). We can also further clarify the result by introducing infinitesimal thickness of the wall and taking approximation around $z=0$, although we cannot determine the shape of the solution within this thickness exactly2.

Now the physical metric can be obtained by multiplying the conformal factor (see eq.(3)) as $d \widetilde{s}^{2}=\mathrm{e}^{2 \beta \phi} d s^{2}$ so that we obtain

$$
d \widetilde{s}^{2}=(1+\widetilde{\kappa}|z|)^{2 \alpha \beta+\alpha^{2}-\frac{1}{2}}\left(-d t^{2}+d z^{2}\right)+(1+\widetilde{\kappa}|z|)^{2 \alpha \beta+1}\left(d x^{2}+d y^{2}\right) .
$$

Since $2 \alpha \beta+1=0$, we have $\omega=0$, then the physical metric becomes nothing but the Minkowski metric

$$
d \widetilde{s}^{2}=-d t^{2}+d z^{2}+d x^{2}+d y^{2}
$$

2 For example, we can cut off the solution for $|z|>\epsilon$ for infinitesimal $\epsilon$. Although we are not able to solve the equations exactly in the region $|z|<\epsilon$ but at least we know $\Phi(0)=0$ and $A, B, \phi$ should be continuous. Then we can check the consistency of the solution eq.(14) at $z=0$ because as $\epsilon \rightarrow 0$ the leading order of the solution should not be much different from this. In fact we only need to use the property $A=B^{\alpha^{2}-1 / 2}$ and $\phi=\alpha \ln B$. Thus it is a good approximation. 
The success of the prescription eq.(12) indeed suggests that we should be able to get thick wall solutions by replacing the step function $\theta(z)$ with a smeared $\theta$-function [13] Thick wall solutions we are really interested in nevertheless should approach to our thin wall solution asymptotically as $|z| \rightarrow \infty$. Also in the the small $\beta$ approximation, if $\beta \rightarrow 0$, $\omega \rightarrow \infty$, and that $\phi \rightarrow 0$, it should recover a thick domain wall in the Einstein gravity case. But solving eq. (13a-e for an arbitrary $\beta$ and a wall thickness seems to be fairly complicated, though it may not be impossible.

\section{Discussion}

Needless to say such a thin dilatonic domain wall is gravitationally stable because it does not carry any gravitational energy-momentum (see eq.(8)). The dilatonic contribution to the energy-momentum tensor precisely cancels the matter part of the energy-momentum tensor to make the space-time flat. It should be important to further look for timedependent or thick wall solutions to check if such a result survives in these cases too.

This result can be very significant in the following sense:

First, such a dilatonic domain wall may not be seriously harmful with respect to the strong CP problem. The domain wall problem in the strong CP problem is generated by the existence of a domain wall that carries too much energy. But the dilatonic domain wall we have at hand does not carry any gravitational energy so that it can exist without ruining the standard cosmology paradigm.

Second, it has been speculated that the extended object in string theory, in particular, the fivebranes [14] [15], can appear as a two-dimensional extended objects in the four-dimensional space-time after compactifications [16]. The metric given in ref. [16] for the sine-Gordon fivebrane is in fact flat because the curvature vanishes. Since the dilaton gravity is a natural gravitational theory from the string theory's point of view, the domain wall for this theory, if it ever exists, may be in such a form. Domain walls in string theory context were also formerly studied in other aspects]17 so that it would be interesting to further study with such an expectation presented here.

From such a point of view one may be tempted to speculate that there may not be a domain wall problem in string theory, although a domain wall exists. Since our conclusion can be applied to a possible thick domain wall case far away from the wall, if such a domain wall exists, say, near the horizon of the universe, we certainly cannot observe any gravitational effects due to the wall. 


\section{Acknowledgements}

The author would like to thank A. Vilenkin for very helpful discussions and C. Vafa

for his hospitality at Harvard, while this work was done. This work was supported in part by NSF grant PHY89-07887 and a World Laboratory Fellowship. 


\section{References}

[1] For reviews, see A. Vilenkin, Phys. Rep. 121 (1985) 263.

[2] For recent reviews in string theory case, see M.J. Duff and J.X. Lu, Class. Quant. Grav. 9 (1992) 1; C.G. Callan, J.A. Harvey and A. Strominger "Supersymmetric String Solitons," Chicago preprint, EFI-91-66 (1991).

[3] E.W. Kolb and M.S. Turner, " The Early Universe," (Addison-Wesley, New York, 1989).

[4] D. La and P. Steinhardt, Phys. Rev. Lett. 62 (1989) 376.

[5] C. Gundlach and M.E. Ortiz, Phys. Rev. D42 (1990) 2521.

[6] J. Scherk and J.H. Schwarz, Nucl. Phys. B81 (1974) 118;

E.S. Fradkin and A.A. Tseytlin, Nucl. Phys. B261 (1985) 1;

C.G. Callan, D. Friedan, E. Martinec and M.J. Perry, Nucl. Phys. B262 (1985) 593.

[7] T.D. Lee, Phys. Rep. 9 (1974) 143.

[8] Ya.B. Zel'dovich, I.Yu. Kobzarev, and L.B. Okun, JETP 40 (1975) 1.

[9] A. Vilenkin, Phys. Rev. D23 (1981) 852.

[10] S. Weinberg, "Gravitation and Cosmology," (Wiley, New York, 1972).

[11] A.H. Taub, Phys. Rev. 103 (1956) 454.

[12] W. Israel, Nuo. Cim. 44B (1966) 1; R. Geroch and J. Traschen, Phys. Rev. D36 (1987) 1017.

[13] H.S. La, to be published.

[14] M.J. Duff, Class. Quan. Grav. 5 (1988) 189;

M.J. Duff, in Superworld II, ed. by A. Zichichi (Plenum, New York, 1990).

[15] A. Strominger, Nucl. Phys. B343 (1990) 167.

[16] H.S. La, "Solitons reduced from Heterotic Fivebranes," CTP-TAMU-36/92 (1992).

[17] K. Choi and J.E. Kim, Phys. Rev. Lett. 55 (1985) 2637;

J.A. Casas and G.G. Ross, Phys. Lett. B198 (1987) 461;

M. Cvetic, F. Quevedo and S.J. Rey, Phys. Rev. Lett. 67 (1991) 1836. 